expense of tissue immunity, so that the last stage of the patient becomes worse than the first.

The following is the method of treatment with tuberculin which I have adopted.

\section{At the Dispensary.}

(a) A course of treatment with T.O.A. (exotoxic) until a certain degree of tolerance is produced.

The following doses are given every eighth day:

0.00001 c.cm., 0.00002 c.cm., 0.00003 c.cm., 0.00004 c.cm., 0.0003 c.cm., 0.0004 c.cm., 0.0005 c.cm., 0.00075 c.cm., 0.001 c.cm.;

(b) When the dose of $0.001 \mathrm{c.cm}$. has been reached a course of treatment with tuberculin T.R. or new tuberculin W., both of which contain endotoxin and exotoxin of the human bacillus, is commenced. The cost of tuberculin T.R. is 8s. 6d. per c.cm., and the cost of new tuberculin W. 6s. per c.cm. The filtrates of the tubercle bacillus are much cheaper, and it is unfortunate that our views regarding the value of tuberculin preparations are no doubt influenced by their price. I employ new tuberculin W. in the following doses:

Immediately following 0.001 c.cm. T.0.A., 0.00005 c.cm., 0.000075 c.cm., 0.0001 c.cm., 0.0002 c.cm., 0.0003 c.cm., 0.0004 c.cm., 0.0005 c.cm., 0.00075 c.cm., 0.001 c.cm., 0.002 c.cm. 0.003 c.cm., 0.004 c.cm., 0.005 c.cm., 0.0075 c.cm., 0.01 c.cm., $0.02 \mathrm{c} . \mathrm{cm} ., 0.03 \mathrm{c.cm} .0 .04 \mathrm{c.cm} ., 0.05$ c.cm., $0.075 \mathrm{c.cm} ., 0.1 \mathrm{c.cm}$, This is the maximum dose, and it is repeated at gradually increasing in tervals.

The above course of treatment applies to well-marked types of the disease with evidence of slight phases of recurring or persistent auto-inoculation. I have not found it necessary or desirable to go above the maximum dose of $0.1 \mathrm{c.cm}$. of pure tuberculin.

\section{In the Sanatorium.}

In the sanatorium a course of treatment with exotoxic tuberculin is replaced in the majority of cases by treatment with induced auto-inoculation. The patient with an active tuberculous focus is thus led from a period of rest through the various stages of induced auto-inoculation to a full course of treatment with tuberculin containing both the endotoxin and exotoxin of the human bacillus. Part of this course will be commenced at the sanatorium, but it will usually be completed at the dispensary.

Elsewhere ${ }^{2}$ I have described the excellent results obtained by a course of treatment with tuberculin T.R. following treatment' with auto-inoculation, and I recom. mend in the sanatorium a course of new tuberculin W. in the following doses:

0.00001 c.cm., 0.00002 c.cm., 0.00003 c.cm., 0.00004 c.cm., 0.00005 c.cm., 0.000075 c.cm., 0.0001 c.cm., 0.0002 c.cm., 0.0003 c.cm., 0.0004 c.cm., 0.0005 c.cm., 0.00075 c.cm., 0.001 c.cm., 0.002 c.cm., 0.003 c.cm., 0.004 c.cm., 0.005 c.cm., 0.0075 c.cm., 0.01 c.cm., 0.02 c.cm., 0.03 c.cm., 0.04 c.cm., 0.05 c.cm., 0.075 c.cm., 0.1 c.cm. The maximum dose to be repeated at gradually increasing intervals.

It is desirable that, in the various districts which embrace sanatorium and dispensary in the scheme for dealing with tuberculosis, there should be some uniformity with regard to the method of administering tuberculin in the sanatorium and dispensary, so that when the patient passes from the sanatorium to the after-care of the dispensary there should be no marked cleavage in the methods of treatment.

\section{Conclusions.}

1. It is desirable that the various districts in which the machinery for the treatment of tuberculosis is being established a uniform system of treatment with tuberculin should be adopted.

2. It is especially desirable that the system of tuberculin treatment carried out in the sanatorium should correspond with the method adopted at the dispensary.

3. A short course of treatment with T.O.A., followed by a course of treatment with new tuberculin W., should form the basis of treatment at the dispensary.

4. A course of treatment by auto-inoculation followed by a course of treatment with new tuberculin W. or tuberculin T.R. should form the basis of treatment in the sanatorium.

\section{PROGNOSIS IN PULMONARY TUBBERCULOSIS: THE RESULTS OBTAINED BY ARNETH'S METHOD.}

By J. B. H. HOLROYD, M.R.C.S.EnG., L.R.C.P.Lond., LATE ASSISTANT MEDICAL OFFICER, SHEFFIELD ENION HOSPITAL.

The prognosis in pulmonary tuberculosis is of vital importance both to the patient individually and to the health of a community. But to make a correct prognosis is in many cases very difficult, and in some almost impossible if physical diagnostic signs are the only guides available.

Arneth's method of blood counting offers, however, some solution of the difficulties encountered in this connexion. When he devised this method he took the neutrophilic leucocyte, which may have I, II, III, IV, or V nuclei, as the cell, and on this he made his count. He then assumed that the cells with I or II nuclei were less resistant than the cells with III, IV, or V nuclei, and that when those less resistant cells were present in large numbers the severity of the case varied directly as the number of these cells.

I adopted this method at the Sheffield Union Hospital, and applied it to 30 cases extending over a period of three months. The observations made on these cases suggested that Arneth was right, and that the prognosis varied inversely as the increase in neutrophiles having I or II nuclei.

The technique employed is as follows:

The specimen of blood is obtained in the usual way by pricking the finger and spreading the drop of blood thus obtained on a clean slide by means of another slide held at an angle of 45 degrees. The slides should be kept in a mixture of equal parts of ether and alcohol, and when required for use are wiped dry with a soft duster. The film of blood thus prepared is allowed to dry in the air, after which staining is proceeded with.

The stain used varies with different observers, and in this particular series of cases Jenner's stain was employed throughout. The slide is flooded with stain, which is kept on for five minutes and then washed off with water, dried and mounted in minutes and then washed off with water, dried and mounted in xylol balsam, care being taken to have an adequate quantity of
stain to obviate the danger of the slide becoming dry. The stain to obviate the danger of the slide becoming dry. The slide is now examined microscopically, and $x^{2}$ in. oil immersion
lens is used. If a larger lens is substituted it is very difficult lens is used. If a larger lens is substituted it is very difficult to be sure of the number of nuclei in the cells. A movable stage should also be used in order to alter the position of the
slide as easily as possible so as to avoid the accident of counting the same field twice; 100 neutrophilic leucocytes are then counted and classified according to the number of their nuclei.

The count is then compared with the normal one observed by Arneth, which has been used as the standard in all the results of this series of cases. The normal is ag follows :

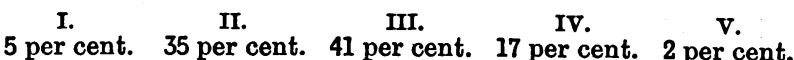

It is noticed that in an unfarourable case there is an increase in the number of cells with I or II nuclei, and usually both I and II are increased; in other words there is a drift to the left. This drift has been present in all cases in this series.

The following examples are given :

1. A. P., aged 33. Physical signs definite and the patient ill. Tubercle bacilli numerous. The count showed on June 14th, 1911:

II.

III.

IV.

V.

44 per cent. 31 per cent. 20 per cent. 5 per cent. 0 per cent. Death occurred on August 10th, 1911.

2. P. R., aged 55. Patient ill. Cavitation present; tubercle bacilli also present. Count on June 19th, 1911, showed :

I. III. IV. V.

45 per cent. 40 per cent. 12 per cent. 2 per cent. 1 per cent. Death on Jane 22nd, 1911.

3. G. E., aged 39. Patient ill, signs definite, and tuberclo bacilli present. Count on June 26th showed :

I.

II.

III.

IV.

V. .

Death on July lst, 1911. 
4. A. H., aged 45. Signs definite ; tubercle bacilli present.

I. II. III. IV.

51 per cent. 39 per cent. 8 per cent. 2 per cent. 0 per cent. Death took place in four days after the count had been taken. 5. J. G., aged 28. Patient ill ; signs definite.

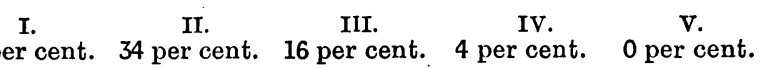
Death in ten days after the count.

6. B. J. G., aged 15. Signs definite and ischio-rectal abscess present, tuberculous in origin. Count taken June 14th :

I. II. III. IV.

33 per cent. 35 per cent. 25 per cent. 5 per cent. 2 per cent.

On September 8th patient appeare! well. No temperature; abscess healed. Count gave:

I. 12 per cent. 40 per cent. 42 per cent. 4 per cent. 2 per cent. III. $\begin{array}{llccc}\text { I. } & \text { II. } & \text { III. } & \text { IV. } & \text { V. } \\ & \text { per cent. } & 40 \text { per cent. } & 42 \text { per cent. } & 4 \text { per cent. } 2 \text { per cent. }\end{array}$ $\begin{array}{llccc}\text { I. } & \text { II. } & \text { III. } & \text { IV. } & \text { V. } \\ & \text { per cent. } & 40 \text { per cent. } & 42 \text { per cent. } & 4 \text { per cent. } 2 \text { per cent. }\end{array}$ As the condition improved the drift to the left was not quite so marked.

The conclusions arrived at after the above observations were that in cases with an unfavourable aspect the cells with I or II or I and II nuclei were present in greater numbers than normally, and that as the improvement of the general condition takes place the drift to the left of the picture is not so marked; or in other words, the cells with I and II nuclei are not so numerous, and approach nearer the normal.

\section{THE EPIGLOTTIC SUTURE: ITS VALUE IN INDIREC' LARYNGOSCOPY *}

\section{By CYRIL HORSFORD, M.D., F.R.C.S.,} LONDON.

THE object of this brief communication is to draw the attention of the members of this Congress to the fact that the field of vision obtained by indirect laryngoscopy may be greatly improved by the passage of a suture through the epiglottis, and that this suture may now be rapidly and safely passed. The suture is recommended as a substitute, more efficient in all respects than the many forms of epiglottis lifters introduced to facilitate intralaryngeal inspection.

I am fully conscious of the enormous boon the direct method has become to laryngeal surgery, and many enthusiasts have entirely given up the indirect method for it. The time is therefore opportune for bringing forward a plea for the revival of the indirect method, supplemented by the suture.

The method is not new, but is a resuscitation of an old idea. I admit that in my first publication on this subject 1 I described it as original, as I could not at that time find any evidence to the contrary. Sir Felix Semon, however, drew my attention to previous work in this field when I demonstrated it at the meeting of the Laryngological Section of the Royal Society of Medicine of London in December, 1907.2

I propose to make no reference to the many varieties of instrumental epiglottic retractors which require the continuous use of the hand to hold the instrument in position. Whether that hand be one of the operator's or that of his assistant is immaterial, for such a method is obviously unsuitable or inconvenient in intralaryngeal operations.

Türck ${ }^{3}$ published in 1866 a description of two instruments which he devised for the purpose of utilizing a thread as an epiglottis lifter. With the first a small hook with a thread attached, was inserted into the epiglottis, but the thread did not penetrate the epiglottis. With the second, however, a needle and thread were passed through the epiglottis and the thread was held by an assistant. This instrument was complicated and its use extremely difficult, largely owing to the fact that local anaesthesia by cocaine was not in use at that time. He further feared that the thread would tear through the epiglottis-a complication which can only be caused by

* Read at the Third Rhino-Laryngological Congress in Berlin, great local unrest. It is no wonder, therefore, that the method fell into disuse. The great value of the principle was, however, recognized, and the instrument was modified and simplified by Schrötter and Tobold. ${ }^{4}$

Jurasz and Jelenffy ${ }^{5}$ recommended the suturing of the glosso-epiglottic ligament instead of the epiglottis, and by this they lifted the epiglottis. Sir Felix Semon and Sir Victor Horsley, ${ }^{6}$ in a series of experiments on dogs, passed a thread through the epiglottis in order to see better the interior of the larynx.

Dr. Gleitsman ${ }^{7}$ of New York has devised an instrument which, from the illustration, appears to be very similar in principle to my latest pattern, and he has found it of great value in rendering possible both a correct diagnosis and an intralaryngeal operation.

The idea of using an epiglottic suture first occurred to me in July, 1906, when I adopted it in order to facilitate an operation of exceptional difficulty-namely, the excision of a very tough fibrous web which united the anterior halves of the vocal cords and extended some way down into the cavity of the larynx. The epiglottis was not overhanging, but $I$ wished to get it out of the way because I was using a sharp-pointed Heryng's knife, followed by sharp punch forceps, and it was necessary to get the best possible view. I used a pair of Spencer Wells forceps, curved at the point, with which I held a small curved and threaded needle. With the aid of the laryngeal mirror the needle was pushed through the epiglottis from before backwards, the point being then caught by the forceps and drawn through. There was not much difficulty in passing the suture and not the slightest discomfort to the patient, who had already b e en thoroughly cocainized in preparation for the operation.

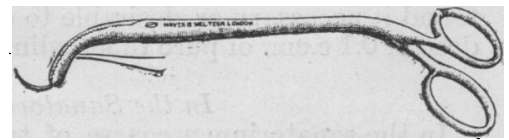

Fig. 1.
In the following year I used a needle-holder made to my design by Messrs. Mayer and Meltzer (see Fig. 1), its principle and use being as above described. This instru ment was shown, and the method described and demonstrated on a patient, at the meeting of the Laryngological Section of the Royal Society of Medicine already referred to. ${ }^{2}$ Soon after I still fur'jher simplified the method by

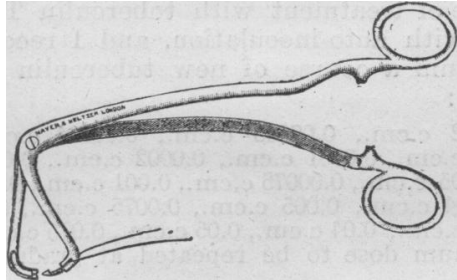

Fig. 2. the suture forceps shown in Fig. 2, which was made for me by Messrs. Mayer and Meltzer. It is a modification of Kurz's forceps used for the passage of deep sutures in pelvic sur. gery. This instrument and its use were demonstrated at another meeting in April, 1908.8 The mechanism of the catch greatly simplified the passage of the suture. Though I have used it with complete success in a large number of cases, I have again modified and simplified it, and my latest pattern, which I call the epiglottic suture forceps No. 2, is the instrument which I now

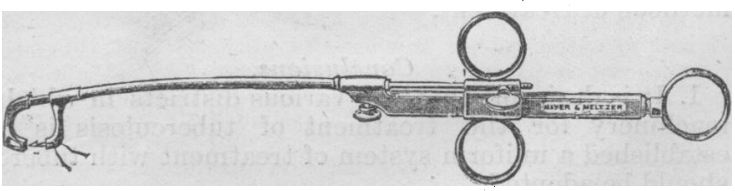

Fig. 3.

recommend (see Fig. 3) ; it may be obtained from Messus. Mayer and Meltzer or H. Pfau, Berlin.

The successful use of either pattern depends, as in other intralaryngeal operations, upon the efficient preparation of the patient for local anaesthesia. Cocaine should be applied to both surfaces of the epiglottis and to the centre of the root of the tongue. It is as well also to apply a little adrenalin to the epiglottis to prevent the possibility of haemorrhage obscuring the field of operation. The needle is first threaded with a long piece of silk and placed in the proximal blade, and the whole carefully sterilized. With the aid of the laryngeal mirror, the 